BULLETIN (New Series) OF THE

AMERICAN MATHEMATICAL SOCIETY

Volume 41, Number 2, Page 149

S 0273-0979(04)01006-7

Article electronically published on January 8, 2004

\title{
CURRENT EVENTS SESSIONS
}

\author{
DAVID EISENBUD
}

Will the Poincaré Conjecture be solved this week? What is the Geometric Langlands Conjecture about? What could one do to analyze a stream of data flowing by too fast to capture? Asking such questions is part of being a mathematician, provoked by our sense that underneath the vastness of mathematics is a fundamental unity that allows us to look into many different corners - though we couldn't possibly work in all of them - as well as by simple curiosity and common-room gossip.

The idea behind the Current Events Session at the Joint Mathematics Meetings, begun last year, was to create an event where the speakers were expected not to report on their own work but to survey some of the most interesting current developments in mathematics, pure and applied. The wonderful tradition of the Bourbaki Seminar was an inspiration, but I hoped for an even wider range of subjects. A broadly constituted advisory committee was charged to select topics and excellent speakers. The programs of the sessions in January 2003 and 2004 will give an idea of the sessions.

2003:

- Michael J. Hopkins: Homotopy theory of schemes

- Ingrid Daubechies: Sublinear algorithms for sparse approximations with excellent odds

- Edward Frenkel: Recent advances in the Langlands Program

- Daniel Tataru: The wave maps equation

\section{4:}

- Margaret H. Wright: The interior-point revolution in optimization: History, recent developments, and lasting consequences

- Thomas C. Hales: What is motivic integration?

- Andrew Granville: It is easy to determine whether or not a given integer is prime

- John W. Morgan: Perelman's recent work on the classification of 3-manifolds

A written exposition greatly increases the number of people who can enjoy the product of these sessions, so all speakers were asked to produce something for distribution. Don Saari agreed to consider these for the Bulletin, and the papers in this issue are the first to be ready. I hope that this is the beginning of a long tradition-but whether or not it is, I hope you enjoy reading these reports!

Mathematical Sciences Research Institute, 1000 Centennial Drive, Berkeley, CaliFORNIA 94720-5070

E-mail address: de@msri.org, eisenbud@math.berkeley.edu

(C)2004 American Mathematical Society 